\title{
ON A CONNECTION BETWEEN THE GENERALIZED INCOMPLETE GAMMA FUNCTIONS AND THEIR EXTENSIONS
}

\author{
M. ASLAM CHAUDHRY' and S.M. ZUBAIR ${ }^{2}$
}

(Received 21 November 1994; revised 2 September 1995)

\begin{abstract}
In this paper we have proved that the generalized incomplete gamma functions and their extensions are mutually related through integral and differential representations.
\end{abstract}

\section{Introduction}

Chaudhry and Zubair considered the generalized gamma functions [4]

$$
\begin{aligned}
& \gamma(\alpha, x ; b)=\int_{0}^{x} t^{\alpha-1} e^{-t-b / t} d t, \\
& \Gamma(\alpha, x ; b)=\int_{x}^{\infty} t^{\alpha-1} e^{-t-b / t} d t,
\end{aligned}
$$

found useful in a variety of transient heat conduction problems $[4,5,13,14]$.

The extensions

$$
\begin{aligned}
& \gamma_{\nu}(\alpha, x ; b)=\left(\frac{2 b}{\pi}\right)^{1 / 2} \int_{0}^{x} t^{\alpha-\frac{3}{2}} e^{-t} K_{\nu+\frac{1}{2}}(b / t) d t \\
& \Gamma_{\nu}(\alpha, x ; b)=\left(\frac{2 b}{\pi}\right)^{1 / 2} \int_{x}^{\infty} t^{\alpha-\frac{3}{2}} e^{-t} K_{v+\frac{1}{2}}(b / t) d t \quad(b>0, x>0,-\infty<\alpha<\infty)
\end{aligned}
$$

of the generalized incomplete gamma functions (1) - (2) were introduced in connection with the generalization of the inverse Gaussian distribution [6]. It is to be noted that

$$
\begin{aligned}
& \Gamma_{0}(\alpha, x ; b)=\Gamma(\alpha, x ; b), \quad \text { and } \\
& \gamma_{0}(\alpha, x ; b)=\gamma(\alpha, x ; b) .
\end{aligned}
$$

${ }^{1}$ Mathematical Sciences Department, King Fahd University of Petroleum and Minerals, Dhahran 31261 , Saudi Arabia

${ }^{2}$ Mechanical Engineering Department, King Fahd University of Petroleum and Minerals, Dhahran 31261 , Saudi Arabia

(C) Australian Mathematical Society, 1997, Serial-fee code 0334-2700/97 
Some applications of the functions (3) - (4) to the representation of Laplace and $K$-transforms were shown in [6]. Several properties of these functions including decomposition formulae, recurrence relations and special cases were also discussed. It was shown that when $v=n$ is an integer, the functions (3) - (4) can be simplified in terms of the generalized incomplete gamma functions (1) - (2). As a matter of fact, it was shown that

$$
\Gamma_{n}(\alpha, x ; b)=\sum_{m=0}^{n} \frac{(2 b)^{-m}}{m !} \frac{\Gamma(n+m+1)}{\Gamma(n-m+1)} \Gamma(\alpha+m, x ; b) .
$$

For nonintegral values of $v$ we were not able to develop a relationship between the functions (1) - (2) and (3) - (4) and it was left as an open problem. The present paper is a continuation of our earlier work $[4,6]$.

In this paper we have found interesting relationships between the functions (1) - (2) and their extensions (3) - (4) for nonintegral values of $v$. Following Erdélyi [8, 9], we shall define the Laplace, Hankel and $K$-transforms of a function $f(t) \quad(0<t<\infty)$ respectively as

$$
\begin{aligned}
L\{f(t) ; s\} & =\int_{0}^{\infty} e^{-s t} f(t) d t \\
H_{\nu}\{f(t) ; y\} & =\int_{0}^{\infty} f(t) J_{\nu}(y t)(y t)^{1 / 2} d t \\
R_{\nu}\{f(t) ; y\} & =\int_{0}^{\infty} f(t) K_{\nu}(y t)(y t)^{1 / 2} d t .
\end{aligned}
$$

\section{Some preliminaries}

In this section we recall some results from [6].

THEOREM 2.1. Let $H(t)=\left\{\begin{array}{ll}1 & \text { if } t>0 \\ 0 & \text { if } t<0\end{array}\right.$ be the Heaviside unit step function and

$$
f(t)=t^{-\alpha-1} e^{-b / t} H\left(t-\frac{1}{x}\right) \quad b>0, x>0 .
$$

Then

$$
R_{\nu}\{f(t) ; y\}=\left(\frac{\pi}{2}\right)^{1 / 2} b^{-\alpha} \gamma_{\nu-\frac{1}{2}}(\alpha, b x ; b y)
$$

and

$$
L\left\{t^{-\alpha-1} e^{-b / t} H\left(t-\frac{1}{x}\right) ; y\right\}=b^{-\alpha} \gamma(\alpha, b x ; b y) \quad(b>0, x>0) .
$$


THEOREM 2.2. Let

$$
f(t)=t^{-\alpha-1} e^{-b / t} H\left(\frac{1}{x}-t\right) H(t) \quad(b>0, x \geq 0, t>0) .
$$

Then

$$
R_{\nu}\{f(t) ; y\}=\left(\frac{\pi}{2}\right)^{1 / 2} b^{-\alpha} \Gamma_{\nu-\frac{1}{2}}(\alpha, b x ; b y)
$$

and

$$
L\left\{t^{-\alpha-1} e^{-b / t} H\left(\frac{1}{x}-t\right) H(t) ; y\right\}=b^{-\alpha} \Gamma(\alpha, b x ; b y) .
$$

\section{Integral representations}

According to (7), the extension $\Gamma_{v}(\alpha, x ; b)$ can be simplified in terms of the generalized gamma functions $\Gamma(\alpha, x ; b)$ for integral values of $\nu$. In this section we shall prove that these functions are related to each other through the integral representations for all $v>-1$. Some special cases of these results are found interesting.

THEOREM 3.1.

$$
\Gamma_{v}(\alpha, x ; y)=\frac{2^{-\nu} y^{-v}}{\Gamma(\nu+1)} \int_{y}^{\infty}\left(\xi^{2}-y^{2}\right)^{\nu} \Gamma(\alpha-\nu-1, x ; \xi) d \xi, \quad(y \geq 0, \nu>-1) .
$$

PROOF. Let

$$
f(t)=t^{-\alpha-1} e^{-1 / t} H\left(\frac{1}{x}-t\right) H(t)
$$

Then, according to (15),

$$
R_{\nu}\{f(t) ; y\}=\left(\frac{\pi}{2}\right)^{1 / 2} \Gamma_{\nu-\frac{1}{2}}(\alpha, x ; y) .
$$

Moreover, according to (16) we have

$$
L\left\{t^{\frac{1}{2}+v} f(t) ; \xi\right\}=\Gamma(\alpha-v-1 / 2, x ; \xi) .
$$

However, according to $[9$, p. 122$]$

$$
R_{\nu}\{f(t) ; y\}=\frac{\pi^{1 / 2} 2^{-v} y^{\frac{1}{2}-v}}{\Gamma\left(\frac{1}{2}+v\right)} \int_{y}^{\infty}\left(\xi^{2}-y^{2}\right)^{\nu-\frac{1}{2}} L\left\{t^{\frac{1}{2}+v} f(t) ; \xi\right\} d \xi \quad \operatorname{Re} \nu>-\frac{1}{2}
$$


And from (19) - (21)

$$
\left(\frac{\pi}{2}\right)^{1 / 2} \Gamma_{\nu-\frac{1}{2}}(\alpha, x ; y)=\frac{\pi^{1 / 2} 2^{-\nu} y^{\frac{1}{2}-\nu}}{\Gamma\left(\frac{1}{2}+\nu\right)} \int_{y}^{\infty}\left(\xi^{2}-y^{2}\right)^{\nu-\frac{1}{2}} \Gamma(\alpha-\nu-1 / 2, x ; \xi) d \xi .
$$

Multiplying both sides in (22) by $\left(\frac{2}{\pi}\right)^{1 / 2}$ and replacing $v$.by $v+\frac{1}{2}$ completes the proof.

COROLLARY 3.1.

$$
\Gamma(\alpha, x ; y)=\int_{y}^{\infty} \Gamma(\alpha-1, x ; \xi) d \xi \quad y \geq 0 .
$$

PROOF. This follows from (17) when $v=0$. It should be noted that (23) can be proved directly from the definition (2). In particular, when $y=0$ in (23) an interesting relation

$$
\Gamma(\alpha, x)=\int_{0}^{\infty} \Gamma(\alpha-1, x ; \xi) d \xi
$$

between the classical incomplete gamma function $\Gamma(\alpha, x)$ and the generalized gamma function $\Gamma(\alpha-1, x ; \xi)$ is found. Several special cases of (24) can be listed. For example, the substitution $\alpha=0$ leads to

$$
-\operatorname{Ei}(-x)=\int_{0}^{\infty} \Gamma(-1, x ; \xi) d \xi
$$

while the substitution $\alpha=1 / 2$ leads to (cf. [4])

$$
\int_{0}^{\infty}\left[e^{-2 \sqrt{\xi}} \operatorname{Erfc}\{\sqrt{x}-\sqrt{\xi / x}\}-e^{2 \sqrt{\xi}} \operatorname{Erfc}\{\sqrt{x}+\sqrt{\xi / x}\}\right] \frac{d \xi}{\sqrt{\xi}}=2 \operatorname{Erfc}(\sqrt{x}) .
$$

THEOREM 3.2.

$$
\gamma_{\nu}(\alpha, x ; y)=\frac{2^{-v} y^{-v}}{\Gamma(\nu+1)} \int_{y}^{\infty}\left(\xi^{2}-y^{2}\right)^{v} \gamma(\alpha-v-1, x ; \xi) d \xi \quad(y \geq 0, \nu>-1) .
$$

PROOF. This is similar to the proof of (17). In particular, substituting $v=0$ in (27), we get

$$
\gamma(\alpha, x ; y)=\int_{y}^{\infty} \gamma(\alpha-1, x ; \xi) d \xi
$$

which can be verified directly from (1).

The substitution $y=0$ and $\alpha=1 / 2$ in (28) leads to

$$
\int_{0}^{\infty} \gamma(-1 / 2, x ; \xi) d \xi=\sqrt{\pi} \operatorname{Erf}[\sqrt{x}]
$$


THEOREM 3.3.

$$
\begin{gathered}
\Gamma_{\nu}(\alpha+\mu, x ; b)=2^{1-\mu}[\Gamma(\mu)]^{-1} b^{\nu+1} \int_{b}^{\infty} \xi^{-\mu-\nu}\left(\xi^{2}-b^{2}\right)^{\mu-1} \Gamma_{\nu+\mu}(\alpha, x ; \xi) d \xi \\
(\nu \geq-1, \mu>0, b>0) .
\end{gathered}
$$

PROOF. Let $f(t)=t^{-\alpha-1} e^{-1 / t} H(1 / x-t) H(t)$. Then, according to (15),

$$
\begin{aligned}
g(y ; \nu)=R_{\nu}\{f(t) ; y\} & =\left(\frac{\pi}{2}\right)^{1 / 2} \Gamma_{\nu-\frac{1}{2}}(\alpha, x ; y), \\
R_{\nu}\left\{t^{-\mu} f(t) ; b\right\} & =\left(\frac{\pi}{2}\right)^{1 / 2} \Gamma_{\nu-\frac{1}{2}}(\alpha+\mu, x ; b) .
\end{aligned}
$$

However (see [9, p. 126(7)]),

$$
\begin{gathered}
R_{\nu}\left\{t^{-\mu} f(t) ; b\right\}=2^{1-\mu}[\Gamma(\mu)]^{-1} b^{\nu+\frac{1}{2}} \int_{b}^{\infty} \xi^{\frac{1}{2}-\mu-\nu}\left(\xi^{2}-b^{2}\right)^{\mu-1} g(\xi ; \nu+\mu) d \xi \\
\left(b>0, \mu>0, \nu>-\frac{1}{2}\right) .
\end{gathered}
$$

From (31) - (33), we get

$$
\Gamma_{\nu-\frac{1}{2}}(\alpha+\mu, x ; b)=\frac{2^{1-\mu} b^{\nu+\frac{1}{2}}}{\Gamma(\mu)} \int_{b}^{\infty} \xi^{\frac{1}{2}-\mu-\nu}\left(\xi^{2}-b^{2}\right)^{\mu-1} \Gamma_{\nu+\mu-\frac{1}{2}}(\alpha, x ; \xi) d \xi .
$$

Replacing $v$ by $v+\frac{1}{2}$ in (34) completes the proof.

\section{COROLLARY 3.2.}

$$
\Gamma(\alpha+\mu, x ; b)=\frac{2^{1-\mu}}{\Gamma(\mu)} \int_{b}^{\infty} \xi^{1-\mu}\left(\xi^{2}-b^{2}\right)^{\mu-1} \Gamma_{\mu-1}(\alpha, x ; \xi) d \xi \quad(\mu>0, b \geq 0) .
$$

PROOF. This follows from (30) when $v=-1$ and the fact that

$$
\Gamma(\alpha, x ; b)=\Gamma_{-1}(\alpha, x ; b)=\Gamma_{0}(\alpha, x ; b) .
$$

In particular, substituting $\mu=1$ in (35), we get

$$
\Gamma(\alpha+1, x ; b)=\int_{b}^{\infty} \Gamma(\alpha, x ; \xi) d \xi,
$$

which can be verified directly from (2).

The substitution $b=0$ in (35) leads to

$$
\Gamma(\alpha+\mu, x)=\frac{2^{1-\mu}}{\Gamma(\mu)} \int_{0}^{\infty} \xi^{\mu-1} \Gamma_{\mu-1}(\alpha, x ; \xi) d \xi \quad \mu>0,
$$

where $\Gamma(\alpha, x)$ is the classical incomplete gamma function. 
THEOREM 3.4.

$$
\begin{aligned}
\gamma_{\nu}(\alpha+\mu, x ; y)=2^{1-\mu}[\Gamma(\mu)]^{-1} y^{\nu+1} \int_{y}^{\infty}\left(\xi^{2}-y^{2}\right)^{\mu-1} \gamma_{v+v}(\alpha, x ; \xi) d \xi \\
(\mu>0, v>-1, y \geq 0) .
\end{aligned}
$$

PROOF. Let

$$
f(t)=t^{-\alpha-1} e^{-1 / t} H\left(t-\frac{1}{x}\right) \quad x>0 .
$$

Then, following the steps of Theorem 3.3, we get the proof of (39). In particular, the substitution $v=-1$ in (39) leads to

$$
\begin{gathered}
\gamma(\alpha+\mu, x ; y)=\frac{2^{1-\mu}}{\Gamma(\mu)} \int_{y}^{\infty} \xi^{1-\mu}\left(\xi^{2}-y^{2}\right)^{\mu-1} \gamma_{\mu-1}(\alpha, x ; \xi) d \xi \\
(x>0, \mu>0, y \geq 0) .
\end{gathered}
$$

\section{Differential representations}

The properties of the $K$-transforms and the relations (11) - (14) could be exploited to prove the differential representations of the generalized incomplete gamma functions and their extensions. In this section we prove these representations.

THEOREM 4.1 .

$$
\begin{aligned}
\gamma_{\nu}(\alpha-m, x ; y)=y^{\nu}\left(-\frac{1}{y} \frac{\partial}{\partial y}\right)^{m}\left[y^{m-v} \gamma_{\nu-m}(\alpha, x ; y)\right] \\
(x>0, m=0,1,2,3, \ldots,) .
\end{aligned}
$$

PROOF. Let $f(t)=t^{-\alpha-1} e^{-1 / t} H(t-1 / x), x>0$. Then, according to (12)

$$
\begin{aligned}
g(y ; v)=R_{\nu}\{f(t) ; y\} & =\left(\frac{\pi}{2}\right)^{1 / 2} \gamma_{\nu-\frac{1}{2}}(\alpha, x ; y) m, \\
R_{\nu}\left\{t^{m} f(t) ; y\right\} & =\left(\frac{\pi}{2}\right)^{1 / 2} \gamma_{\nu-\frac{1}{2}}(\alpha-m, x ; y) .
\end{aligned}
$$

However, according to $[9$, p. 125(4)],

$$
R_{v}\left\{t^{m} f(t) ; y\right\}=y^{v+\frac{1}{2}}\left(-\frac{1}{y} \frac{\partial}{\partial y}\right)^{m}\left\{y^{m-v-\frac{1}{2}} g(y ; v-m)\right\} .
$$


Therefore, from (43) - (45), we get

$$
\gamma_{\nu-\frac{1}{2}}(\alpha-m, x ; y)=y^{\nu+\frac{1}{2}}\left(-\frac{1}{y} \frac{\partial}{\partial y}\right)^{m}\left\{y^{m-\nu-\frac{1}{2}} \gamma_{\nu-m-\frac{1}{2}}(\alpha, x ; y)\right\} .
$$

Replacing $v$ by $v+\frac{1}{2}$ in (46) completes the proof of (42). In particular the substitution $v=m$ in (42) leads to

$$
\gamma_{m}(\alpha-m, x ; y)=y^{m}\left(-\frac{1}{y} \frac{\partial}{\partial y}\right)^{m}\{\gamma(\alpha, x ; y)\} \quad(m=0,1,2,3, \ldots,) .
$$

THEOREM 4.2.

$$
\Gamma_{\nu}(\alpha-m, x ; y)=y^{\nu}\left(-\frac{1}{y} \frac{\partial}{\partial y}\right)^{m}\left\{y^{m-\nu} \Gamma_{\nu-m}(\alpha, x ; y)\right\} \quad(m=0,1,2,3, \ldots,) \text {. (48. }
$$

PROOF. If we take $f(t)=t^{-\alpha-1} e^{-1 / t} H(1 / x-t) H(t)$ and follow the steps of the proof of Theorem (4.1), we get the proof of (48). In particular the substitution $v=m$ in (48) leads to

$$
\Gamma_{m}(\alpha-m, x ; y)=y^{m}\left(-\frac{1}{y} \frac{\partial}{\partial y}\right)^{m}\{\Gamma(\alpha, x ; y)\} \quad(m=0,1,2,3, \ldots,) .
$$

\section{Functional recurrence relations}

THEOREM 5.1.

$$
\gamma_{v}(\alpha+1, x ; y)=\frac{y}{2 v+1}\left[\gamma_{v+1}(\alpha, x ; y)-\gamma_{v-1}(\alpha, x ; y)\right] .
$$

PROOF. Let $f(t)=t^{-\alpha-1} e^{-1 / t} H(t-1 / x), x>0$. Then, according to (12),

$$
R_{v}\left\{t^{-1} f(t) ; y\right\}=\left(\frac{\pi}{2}\right)^{1 / 2} \gamma_{v-\frac{1}{2}}(\alpha+1, x ; y)=g(y ; v) .
$$

However, according to $[9$, p. 125(5)],

$$
R_{\nu}\left\{t^{-1} f(t) ; y\right\}=\frac{y}{2 v}[g(y, v+1)-g(y ; v-1)] .
$$

From (51) - (52), we get

$$
\gamma_{\nu-\frac{1}{2}}(\alpha+1, x ; y)=\frac{y}{2 v}\left[\gamma_{v+\frac{1}{2}}(\alpha, x ; y)-\gamma_{\nu-\frac{3}{2}}(\alpha, x ; y)\right] .
$$

Replacing $v$ by $v+\frac{1}{2}$ in (53) completes the proof.

\section{THEOREM 5.2.}

$$
\Gamma_{\nu}(\alpha+1, x ; y)=\frac{y}{2 v+1}\left[\Gamma_{v+1}(\alpha, x ; y)-\Gamma_{v-1}(\alpha, x ; y)\right] \quad(x \geq 0, y>0) .
$$

PROOF. This is similar to the proof of Theorem 5.1. 


\section{6. $C^{(\nu)}(\alpha, x ; y)$ and $S^{(\nu)}(\alpha, x ; y)$ functions}

The Hankel and $K$-transforms are related to each other via $[9$, p. 121]

$$
H_{\nu}\{f(t) ; y\}=\frac{1}{\pi}\left[e^{\frac{i}{2}\left(\nu+\frac{1}{2}\right) \pi} R_{\nu}\{f(t) ; i y\}+e^{-\frac{i}{2}\left(\nu+\frac{1}{2}\right) \pi} R_{\nu}\{f(t) ;-i y\}\right] .
$$

Taking $f(t)$ as defined by (18), replacing $v$ by $v+\frac{1}{2}$ in (55) and using (15), we get

$$
\begin{aligned}
& H_{\nu+\frac{1}{2}}\left\{t^{-\alpha-1} e^{-1 / t} H\left(\frac{1}{x}-t\right) H(t) ; y\right\}= \\
& \quad \frac{1}{\sqrt{2 \pi}}\left[e^{\frac{i}{2}(\nu+1)} \Gamma_{\nu}(\alpha, x ; i y)+e^{-\frac{i}{2}(v+1)} \Gamma_{\nu}(\alpha, x ;-i y)\right] .
\end{aligned}
$$

Substituting $x=0$ in (56) and using the relation

$$
H(\infty-t)=1,
$$

we get

$$
H_{\nu+\frac{1}{2}}\left\{t^{-\alpha-1} e^{-1 / t} ; y\right\}=\frac{1}{\sqrt{2 \pi}}\left[e^{\frac{i}{2}(\nu+1)} \Gamma_{\nu}(\alpha, 0 ; i y)+e^{-\frac{i}{2}(v+1)} \Gamma_{\nu}(\alpha, 0 ;-i y)\right] .
$$

According to [9, p. 30(15)],

$$
H_{v+\frac{1}{2}}\left\{t^{-3 / 2} e^{-1 / t} ; y\right\}=2 \sqrt{y} J_{v+\frac{1}{2}}(\sqrt{2 y}) K_{v+\frac{1}{2}}(\sqrt{2 y}) .
$$

Substituting $\alpha=\frac{1}{2}$ in (58) and using (59), we get an interesting relation

$$
\begin{gathered}
e^{\frac{i}{2}(v+1)} \Gamma_{v}(1 / 2,0 ; i y)+e^{-\frac{i}{2}(v+1)} \Gamma_{v}(1 / 2,0 ;-i y) \\
=2 \sqrt{2 \pi y} J_{v+\frac{1}{2}}(\sqrt{2 y}) K_{v+\frac{1}{2}}(\sqrt{2 y}) .
\end{gathered}
$$

In particular, for $v=-1$ in $(60)$ and using $\Gamma_{-1}(\alpha, x ; b)=\Gamma(\alpha, x ; b)$, we get

$$
\begin{aligned}
\Gamma(1 / 2,0 ; i y)+\Gamma(1 / 2,0 ;-i y) & =2 \sqrt{2 \pi y} J_{-1 / 2}(\sqrt{2 y}) K_{-1 / 2}(\sqrt{2 y}) \\
& =2 \sqrt{\pi} e^{-\sqrt{2 y}} \cos (\sqrt{2 y}),
\end{aligned}
$$

which can be verified directly from (2). Similarly, the substitution $v=0$ in (60) leads to

$$
e^{\frac{i}{2} \pi} \Gamma(1 / 2,0 ; i y)+e^{-\frac{i}{2} \pi} \Gamma(1 / 2,0 ;-i y)=2 \sqrt{\pi} e^{-\sqrt{2 y}} \sin (\sqrt{2 y}) .
$$


Therefore, it seems natural to introduce a new pair of functions defined by

$$
\begin{aligned}
& C^{(\nu)}(\alpha, x ; y)=\frac{1}{2}\left[e^{i(\nu+1) \pi / 2} \Gamma_{\nu}(\alpha, x ; i y)+e^{-i(\nu+1) \pi / 2} \Gamma_{\nu}(\alpha, x ;-i y)\right], \\
& S^{(\nu)}(\alpha, x ; y)=\frac{1}{2 i}\left[e^{i(\nu+1) \pi / 2} \Gamma_{\nu}(\alpha, x ; i y)-e^{-i(\nu+1) \pi / 2} \Gamma_{\nu}(\alpha, x ;-i y)\right] .
\end{aligned}
$$

The identities $(60)-(62)$ can now be written as

$$
\begin{aligned}
& C^{(v)}(1 / 2,0 ; y)=2 \sqrt{2 \pi y} J_{v+\frac{1}{2}}(\sqrt{2 y}) K_{v+\frac{1}{2}}(\sqrt{2 y}), \\
& C^{(-1)}(1 / 2,0 ; y)=2 e^{-\sqrt{2 y}} \cos (\sqrt{2 y}), \\
& C^{(0)}(1 / 2,0 ; y)=2 e^{-\sqrt{2 y}} \sin (\sqrt{2 y}) .
\end{aligned}
$$

\section{Acknowledgment}

The authors acknowledge the support provided by King Fahd University of Petroleum and Minerals under the research project MS/GAMMA/171. The helpful comments made by the reviewer are appreciated.

\section{References}

[1] M. Aslam Chaudhry and S. M. Zubair, "Analytic study of temperature solutions due to gamma type moving point-heat sources", Int. J. of Heat and Mass Transfer 36 (1991) 1633-1637.

[2] M. Aslam Chaudhry and S. M. Zubair, "Generalized incomplete gamma functions with applications", J. of Comp. and Appl. Math. 55 (1994) 99-124.

[3] M. Aslam Chaudhry and S. M. Zubair, "On an extension of generalized incomplete gamma functions with applications", J. Austral. Math. Soc. Ser. B 37 (1996) 392-405.

[4] A. Erdélyi,et al., Tables of Integral Transforms Vol. I McGraw-Hill, New York (1954).

[5] A. Erdélyi,et al., Tables of Integral Transforms Vol. II McGraw-Hill, New York (1954).

[6] S. M. Zubair and M. Aslam Chaudhry, "Temperature solutions due to continuously operating spherical-surface-heat sources in an infinite medium", Intl. Communications in Heat and Mass Transfers 18 (1991) 805-812.

[7] S. M. Zubair and M. Aslam Chaudhry, "Temperature solutions due to continuously operating, gamma-type heat sources in an infinite medium", Fundamental Problems in Conduction Heat Transfer (ASME-HTD) 207 (1992) 63-68. 